\title{
Primacy and recency in primed free association and associative cued recall
}

\author{
BARBARA M. BROOKS \\ University of East London, London, England
}

\begin{abstract}
Two experiments compared the serial positions of primed words in an implicit free association test with words recalled in a cued recall test. In both tests, weakly or strongly related word pairs were studied, and the first words of each pair formed the test cues. In the implicit test, weakly related words pairs showed primacy and extended recency effects but strongly related word pairs did not. In the explicit test, both weakly and strongly related word pairs showed primacy and extended recency effects. These functional dissociations between implicit and explicit memory tests indicate that strongly related word pairs are encoded together because they have unitized memory representations that function as integrated units without requiring any additional associative links to be made, but that an additional system or process is required to strengthen weakly related word pairs during encoding. In addition, a further additional system or process is accessed by explicit retrieval.
\end{abstract}

Recording the serial position of items recalled has proved to be a useful method of investigating such issues as the distinction between short-term and long-term memory (see, e.g., Glanzer \& Cunitz, 1966), the role of organization in learning (see, e.g., Tulving, 1962), and the extent of rehearsal during study (see, e.g., Rundus, 1971). Given the usefulness of this research method, it is surprising that relatively few studies have investigated whether or not serial position effects occur in implicit memory tests, since this line of inquiry would enhance our knowledge of the systems and processes that contribute to human memory.

Those studies that have so far been performed have all used one implicit memory test, word stem completion, but there is a lack of agreement between them as to whether or not primacy or recency effects occur. For example, Rybash and Osborne (1991) found a significant recency effect but no primacy effect, whereas Gershberg and Shimamura (1994) found transient primacy effects in two of three experiments and a transient recency effect in one of three experiments. It is possible that other factors, such as differences in word frequency, word memorability, or ease of completion of word stems, may have contributed to these different results.

To control for such extraneous factors, Brooks (1994) rotated the presentation of words at study so that each word appeared in every other serial position in Experiment $l$ and in every serial position in Experiment 2. Using this counterbalancing procedure, and testing immediately in the reverse to study order, she did not find either

I am grateful to John Gardiner for his valuable advice in the preparation of this manuscript. I also thank Elliot Hirshman, Kathleen McDermott, and Alan Richardson-Klavehn for their helpful comments. Correspondence should be addressed to B. M. Brooks, University of East London, Psychology Department, Romford Road, London E15 4LZ, England (e-mail: b.m.brooks@uel.ac.uk) primacy or recency effects in word stem completion relative to significant primacy and recency effects in explicit cued recall. These results revealed another dissociation between tests of implicit and explicit memory and provided further evidence that conscious retrieval processes were not involved in this implicit test. However, the study only investigated serial position effects in word stem completion, and priming effects have been shown to vary across different implicit memory tests. For example, variables such as levels of processing, the generation effect, and modality have been found to dissociate performance in different implicit memory tests (see Roediger \& McDermott, 1993, for a review).

Even priming in the same test has been found to respond differently to a levels-of-processing manipulation, depending on the stimulus items used. For example, Schacter and McGlynn (1989, Experiment 3) found that priming of strongly related paired associates (e.g., TABLECHAIR) was independent of a levels-of-processing manipulation that significantly affected explicit cued recall. In contrast, in Experiment 4, they found that priming of unrelated word pairs (e.g., TABLE-KEY) was susceptible to a levels-of-processing study manipulation. The difference in susceptibility to levels of processing between the two free association tests appears to be attributable to the degree of association of the stimulus items. Strongly related word pairs may not be susceptible to different levels of processing because they have "unitized" memory representations that function as integrated units, and activation of unitized stimuli may occur automatically during the study phase (Diamond \& Rozin, 1984; Graf \& Mandler, 1984). Conversely, nonunitized stimuli may be susceptible to different levels of processing because new or stronger associative connections between them are required to be made during encoding.

The presence or absence of levels of processing has proved a useful method of investigation, but the more 
fine-grained analysis of examining the serial positions of studied target items might reveal additional insights into why the degree of association of word pairs affects free association performance. Experiment 1 therefore examined serial position effects in a free association test and a cued recall test using weakly related word pairs as stimuli. If it is the case that an effortful process is required to bind weakly related word pairs during the study phase, this might result in a primacy effect in free association priming of weakly related word pairs: Binding of the first few word pairs studied would benefit from more rehearsal (elaborative or rote) and more focused attention than the remaining word pairs--the current explanations for primacy effects (Atkinson \& Shiffrin, 1968; Glenberg et al., 1980; Rundus, 1971).

A prediction with regard to possible recency effects is complicated because it is difficult to distinguish between recency effects that might reflect forgetting during the course of the study (hitherto referred to as extended recency effects) and recency effects associated with the recency component of the serial position curve (hitherto referred to as short-term recency effects). However, there are two main differences between these two types of recency effect. One is that short-term recency effects generally involve only two or three stimulus items, whereas extended recency effects would be likely to involve all the stimulus items. The second is that short-term recency effects have been found to disappear if a 20-sec interpolated task is inserted between study and test (Glanzer \& Cunitz, 1966), whereas extended recency effects would not be affected by this manipulation to the same extent. Short-term recency effects are not predicted in free association because they are presumed to reflect conscious retrieval of studied items (Craik, 1970; Glanzer \& Cunitz, 1966). However, extended recency effects might be predicted if the encoded associations between weakly related word pairs were susceptible to decay or interference throughout the course of the test.

\section{EXPERIMENT 1}

Experiment 1 investigated whether primacy and extended recency effects would occur in an implicit memory test of free association relative to an explicit test of cued recall. In both tests, weakly related word pairs were studied, and the first words of each pair formed the test cues. The test sheet was designed to capture short-term recency effects by testing studied items in the reverse to studied order. Before performing the implicit test, subjects were informed of the relationship between the study phase and the test but were told to ignore it and write the first word that came to mind to each of the word cues. Informing subjects of this relationship enabled the test to be performed immediately and reduced the possibility that subjects who became aware of the relationship between the study and test words during the test phase might use intentional retrieval strategies (see, e.g., Bowers \&
Schacter, 1990; Richardson-Klavehn, Gardiner, \& Java, 1994; Richardson-Klavehn, Lee, Joubran, \& Bjork, 1994).

It was predicted that both the implicit and explicit tests would show primacy and extended recency effects. In the implicit test, these effects would be attributable to the effortful component required to bind the weakly related word pairs together during encoding: A primacy effect would reflect better binding of the first few word pairs, whereas an extended recency effect might result from interference or decay to the association between the word pairs. In the explicit test, this same effortful component would be involved plus an additional effortful component - that of thinking back to the study phase and trying to remember the studied words.

\section{Method}

Subjects. Students and staff from Goldsmiths College, University of London $(N=144)$, age range $18-52$ years, participated in the experiment. They were randomly allocated to two groups of $72 \mathrm{sub}$ jects and tested individually.

Design and Materials. The design factors were test (implicit vs. explicit) and serial position, with test manipulated between subjects and serial position manipulated within subjects. The stimulus materials consisted of 36 cards. Two weakly related words in which the second word of the pair was produced to the first word an average of 12 times in 1,000 (Minnesota Word Association Norms; Jenkins, 1970) were printed on each card (e.g., RED HAIR, HUNGRY MAN). The 36 cards were randomly allocated to two sets of 18 cards that were counterbalanced so that when one set was presented the other set formed the unstudied target words. Cards within each set were sequentially numbered from 1 to 18 . During the course of the experiment, the sequential order of each set of cards was kept constant but the positional order varied; each set of cards was advanced by one number after it had been presented once in the implicit and once in the explicit memory test. This procedure allowed full counterbalancing of stimuli since every word pair appeared twice in every serial position for each test. The first words from each word pair formed the test cues. They were equally spaced around the circumference of a circle with words from each stimulus set appearing alternately. The test sheet was covered by a cardboard circular mask, fixed at the center, with a three-sided cut-out on the circumference that allowed one word to be viewed at a time. When the mask was rotated in a clockwise direction, subjects saw the first word from each pair of words they had previously studied, in reverse order, interspersed by unstudied words.

Procedure. Subjects were randomly assigned to the implicit or the explicit test group. Both groups received the same preliminary instructions - to try to memorize the pairs of words they were about to see. Eighteen word pairs were then shown to them on cards, one word pair every $3 \mathrm{sec}$. After 1 subject from each group had seen the stimuli in an identical order, the positional order of the cards was advanced by one (while keeping the sequential order the same) before they were presented again. In addition, alternate subjects in each group saw a different set of stimuli. Subjects were tested immediately. Each subject received an identical test sheet, with a mask open at the word immediately preceding the cue word from the last studied word pair, and was instructed to move the mask in a clockwise direction. In the implicit test, subjects were asked to write word associations alongside each of the words on the test sheet. They were instructed not to try to think back to the study cards and to write words from the study cards only if they were the first words that came to mind. If a word did not immediately come to mind, they were asked to leave it blank. In the explicit test, subjects were instructed to study each word on the test sheet. If they remembered 
that the word had appeared on the study cards, they were required to try to recall and write the other member of the word pair alongside the cue word. They were informed of the presence of some unstudied words and were instructed not to guess associations. On completion, subjects in the implicit test were asked whether they had actually written the first words that came to mind or whether they had deliberately tried to recall words from the study episode. Two subjects were replaced because they had misunderstood the test instructions and had deliberately tried to recall words in the free association test.

\section{Results and Discussion}

In all the statistical analyses reported in this study, the alpha level was set at .05 . Mean proportions of studied target items associated/recalled as a function of serial position and test are shown in Table 1 . There was significant priming in the implicit test [studied target word association, $p=.48$; unstudied target word association, $p=.03$; $t(71)=24.23, S E M=0.02]$. Since the level of association of unstudied target words was very low, baseline association should not have masked any possible serial position effects in the implicit test. Similarly, incorrect recall in the explicit test was very low [studied target word recall, $p=.56$; incorrect recall, $p=.05 ; t(71)=22.53, S E M=$ 0.02 ], indicating that guessing should not have masked serial position effects in the explicit test.

To reduce noise in the serial position graphs, data were collapsed across three consecutive serial positions in each test. In addition, the probability of baseline association was subtracted from each data point in the implicit test and the probability of incorrect recall was subtracted from each data point in the explicit test. A graph of these results is shown in Figure 1. Although performance in the explicit test was higher than performance in the im-

Table 1

Probability of Associating/Recalling Studied Target Words as a Function of Serial Position and Test in Experiment 1, and Word Relatedness, Serial Position, and Test in Experiment 2

\begin{tabular}{|c|c|c|c|c|c|c|}
\hline \multirow{3}{*}{$\begin{array}{c}\text { Serial } \\
\text { Position } \\
\end{array}$} & & & \multicolumn{4}{|c|}{ Experiment 2} \\
\hline & \multicolumn{2}{|c|}{ Experiment 1} & \multicolumn{2}{|c|}{ Strongly Related } & \multicolumn{2}{|c|}{ Weakly Related } \\
\hline & Implicit & Explicit & Implicit & Explicit & Implicit & Explicit \\
\hline 1 & .57 & .65 & .53 & .81 & .50 & .67 \\
\hline 2 & .49 & .57 & .56 & .69 & .31 & .67 \\
\hline 3 & .35 & .49 & .56 & .72 & .22 & .67 \\
\hline 4 & .43 & .49 & .61 & .75 & $.3 \mathrm{l}$ & .44 \\
\hline 5 & .36 & .53 & .56 & .67 & .33 & .56 \\
\hline 6 & .36 & .46 & .64 & .64 & .31 & .58 \\
\hline 7 & .54 & .54 & .53 & .53 & .33 & .53 \\
\hline 8 & .47 & .51 & .50 & .58 & .44 & .64 \\
\hline 9 & .36 & 61 & .72 & .69 & .42 & .61 \\
\hline 10 & .46 & 63 & .69 & .56 & .47 & .58 \\
\hline 11 & .50 & .51 & .64 & .67 & .42 & .61 \\
\hline 12 & .60 & .61 & .69 & .72 & .50 & .61 \\
\hline 13 & .42 & .60 & .64 & .64 & .50 & .64 \\
\hline 14 & .61 & .57 & .47 & .67 & .47 & .67 \\
\hline 15 & .50 & .61 & .64 & .61 & .44 & .61 \\
\hline 16 & .61 & .60 & .53 & .78 & .53 & .56 \\
\hline 17 & .50 & .64 & .69 & .64 & .58 & .64 \\
\hline 18 & .60 & .54 & .58 & .72 & .39 & .69 \\
\hline
\end{tabular}

plicit test, both appeared to show primacy and extended recency effects throughout the course of testing.

To test this interpretation of the data, a $2 \times 6$ analysis of variance (ANOVA) with one between-subjects factor, test (implicit vs. explicit), and one within-subjects factor, serial position ( $1-3$ vs. $4-6$ vs. $7-9$ vs. $10-12$ vs. $12-15$ vs. 16-18) was performed on data in which the probabilities of baseline performance and incorrect recall had been subtracted. The main effects of test $[F(1,142)=$ $\left.4.62, M S_{\mathrm{e}}=0.16\right]$ and serial position $[F(5,710)=4.18$, $\left.M S_{\mathrm{e}}=0.08\right]$ were significant, with no interaction between test and serial position $\left[F(5,710)=0.41, M S_{\mathrm{e}}=\right.$ 0.08]. Comparisons between Serial Positions $1-3$ and 4-6 to investigate possible primacy effects showed significant (one-tailed) differences in the implicit test $[t(71)=$ 1.64, $S E M=0.05]$ and in the explicit test $[t(71)=1.83$, $S E M=0.04]$. (These comparisons were performed on adjacent sets of serial positions because of the apparent extended recency effect that occurred during the course of the tests.) As shown in Table 1, both tests appeared to show primacy effects for the first two serial positions, but these effects were attenuated when collapsed across three consecutive serial positions. When the probability of association/recall from Serial Positions 1-2 was compared with the probability of association/recall from Serial Positions 3-6, there were more pronounced primacy effects in the implicit test $[t(71)=3.18, S E M=0.05]$ and in the explicit test $[t(71)=2.25, S E M=0.05]$.

Further comparisons between Serial Positions 13-15 and 16-18 to investigate possible short-term recency effects were not significant in the implicit test $[t(71)=1.44$, $S E M=0.04]$ or in the explicit test $[t(71)=0.00, S E M=$ $0.05]$. (These comparisons were performed on adjacent sets of serial positions because of the apparent extended recency effects that occurred during the course of the tests.) However, a $2 \times 5$ polynomial test of order between test and nonprimacy serial positions (4-6, 7-9, 10-12, 13-15, and 16-19) showed a significant linear trend $\left[F(142)=17.06, M S_{\mathrm{e}}=0.09\right]$, with no interaction between test and nonprimacy serial positions $[F(1,142)=$ $\left.1.20, M S_{\mathrm{e}}=0.09\right]$. Although neither test appeared to show short-term recency effects, both tests showed extended recency effects.

In accordance with the experimental hypothesis, the weakly related word pairs produced a significant two-item primacy effect in both the implicit and the explicit tests. This result contrasted with the results of a previous study, which used the same experimental procedure with word stem completion but did not find any indication of a primacy effect (Brooks, 1994). The main difference between these studies was that only free association of weakly related word pairs required an associative bond to be made between the two stimulus words during the study phase. In the word stem completion test, single word stimuli, in which no associative bond was required to be made, were studied. It is therefore likely that the effortful associative 


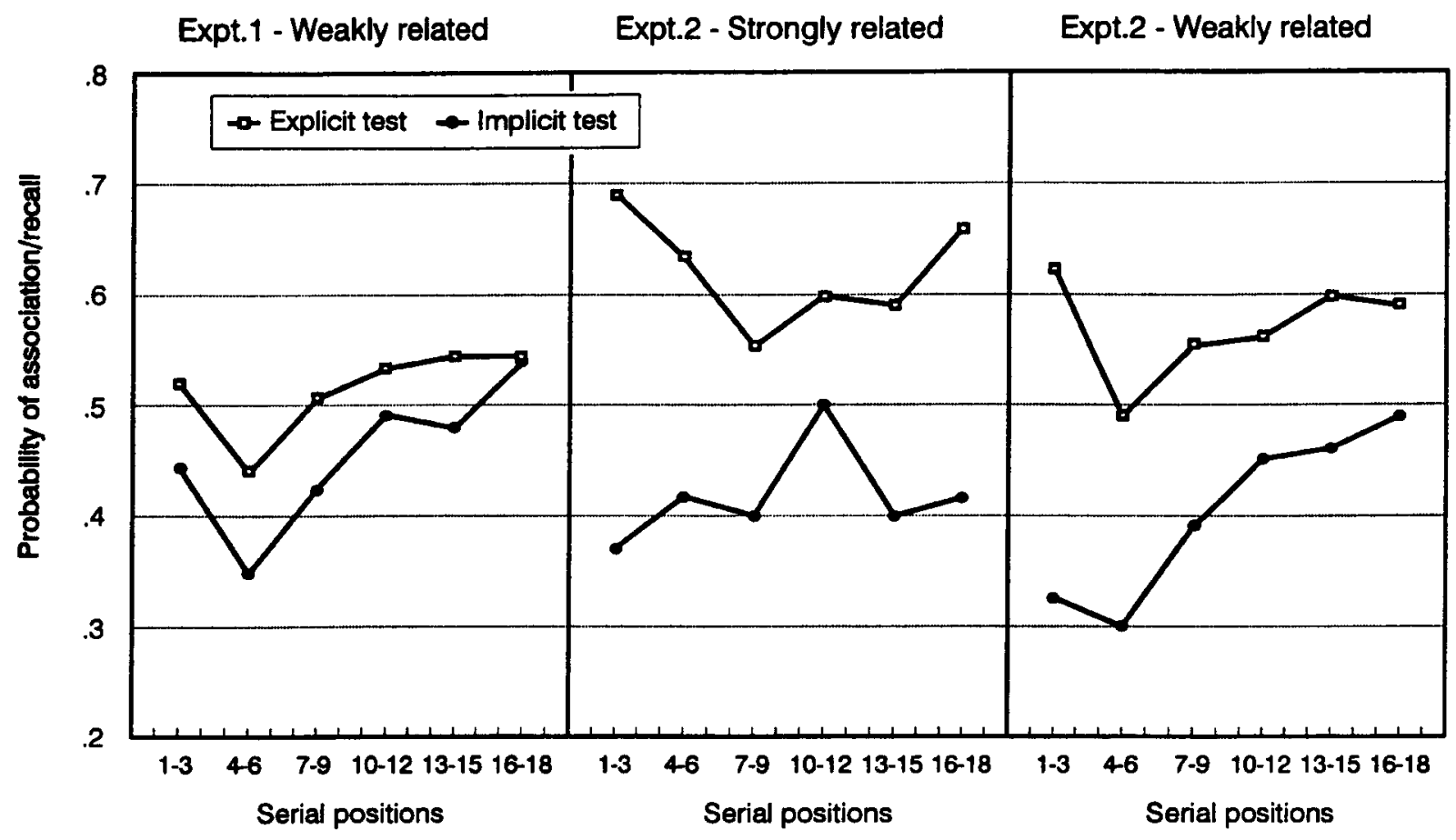

Figure 1. Probability of associating/recalling weakly and strongly related studied target words as a function of serial position and test with the probability of baseline association subtracted in the implicit free association test and the probability of incorrect recall subtracted in the explicit free recall test.

binding component of free association of weakly related word pairs was responsible for the primacy effect that occurred in this test. There was also an extended recency effect throughout the course of the free association test that was not found in the word stem completion test. A possible explanation for this is decay or interference to the encoded connection between the word pairs.

However, these comparisons are between different studies and may not be reliable. It was therefore necessary to test the assumptions within a single experiment. If it is the case that weakly related word pairs require an additional task component during the study phase to strengthen the associative connections between them, word pairs that are already strongly related should not require a similar processing component. Experiment 2 therefore examined serial position effects in free association using both weakly and strongly related word pairs.

\section{EXPERIMENT 2}

Experiment 2 investigated whether differences in the strength of association between weakly and strongly related word pairs would produce differences in primacy and extended recency effects in free association and cued recall. If differences between strongly and weakly related word pairs were found in a single experiment, this would provide more reliable evidence that the effortful process of binding weakly related word pairs together during encoding was responsible for the primacy and extended recency effects found in free association with weakly related word pairs in Experiment 1 . In cued recall, the additional effortful component of trying to remember studied words might result in primacy and extended recency effects, irrespective of the strength of association of the word pair stimuli. Free association and cued recall performance were therefore compared using strongly or weakly related word pairs as stimuli. It was predicted that only weakly related word pairs would show primacy and extended recency effects in the implicit test, whereas both weakly and strongly related word pairs would show primacy and extended recency effects in the explicit test.

\section{Method}

Subjects. Students and staff from Goldsmiths College, University of London, and the University of East London $(N=144)$, age range 18-40 years, participated in the experiment. They were randomly allocated to four groups of 36 subjects and tested individually.

Design and Materials. The design factors were test, word relatedness, and serial position, with test and word relatedness manipulated between subjects and serial position manipulated within subjects. The stimulus materials consisted of 72 cards with two semantically related words printed on each (e.g., SQUARE-CIRCLE, SQUARE-HOLE, ANGER-FEAR, ANGER-DIVORCE). As can be seen from the examples, each initial cue word was followed by either a strongly related word or a weakly related word. Two sets of 36 of these word pairs, one in which the second word of the pair was produced to the first an average of 192 times in 1,000 and the second in which the 
second word was produced to the first an average of 6 times in 1,000, were selected from the Minnesota Word Association Norms (Jenkins, 1970). The set of strongly related word pairs was randomly divided into two further sets of 18 cards, and the weakly related word pairs were divided into two corresponding sets of 18 cards. These two sets of stimuli were numbered and counterbalanced in the same manner as in Experiment 1. This procedure allowed full counterbalancing of stimuli since every word pair appeared once in every serial position for each test. The same test sheet was used for every test. On the test sheet, the shared first words from the word pairs formed the test cues. As in Experiment 1, these words were equally spaced around the circumference of a circle with words from each stimulus set appearing alternately through a mask.

Procedure. The only procedural difference from Experiment 1 was that subjects were randomly allocated to four experimental groups rather than two. The additional experimental groups were necessary so that subjects performed implicit or explicit tests using either strongly or weakly related word pairs.

\section{Results and Discussion}

Mean proportions of studied target items associated/ recalled as a function of serial position, test, and word relatedness are shown in Table 1. There was significant priming in the two implicit tests [strongly related wordsstudied target word association, $p=.61$; unstudied target word association, $p=.17 ; t(35)=11.15, S E M=0.04$; weakly related words-studied target word association, $p=.42$; unstudied target word association, $p=.01$; $t(35)=11.15, S E M=0.04]$. Since unstudied target word association in the two implicit tests was low, baseline association should not have masked any possible serial position effects in the implicit test. Incorrect recall in the two explicit tests was also low [strongly related words-studied target word recall, $p=.67$; incorrect recall, $p=.05$; $t(35)=16.85, S E M=0.04$; weakly related words-studied target word recall, $p=.61$; incorrect recall, $p=.04$; $t(35)=15.47, S E M=0.04]$, indicating that guessing should not have masked serial position effects in the explicit tests.

To reduce noise in the serial position graphs, data were collapsed across three consecutive serial positions in each test. In addition, the probability of baseline association (strongly related words, $p=.17$; weakly related words, $p=.01$ ) was subtracted from each data point in the implicit tests and the probability of incorrect recall (strongly related words, $p=.05$; weakly related words, $p=.04$ ) was subtracted from each data point in the explicit test. Graphs of these results are shown in Figure 1. It appears from Table 1 and Figure 1 that strongly related word pairs produced a primacy effect in the explicit test but not in the implicit test. Conversely, Table 1 indicates that weakly related word pairs produced primacy effects in both the implicit and the explicit tests. It should be noted, however, that the apparent single item primacy effect in the weakly related implicit test in Table 1 is not evident in Figure 1, in which three consecutive serial positions are collapsed into one. The extended recency effect in the implicit test appears to be steeper for weakly related than for strongly related word pairs. In the statistical analysis of the data, strongly and weakly related word pairs were analyzed separately because of differences in baseline completion.

Strongly related word pairs. A $2 \times 6$ ANOVA with one between-subjects factor, test, and one within-subjects factor, serial position, was performed to calculate whether there was a significant difference between serial positions in the explicit and implicit tests. Probabilities of baseline performance and incorrect recall were subtracted from each data point prior to the analysis. The main effect of test was significant $\left[F(1,70)=16.57, M S_{\mathrm{e}}=0.25\right]$, but not the main effect of serial position $[F(5,350)=0.68$, $\left.M S_{\mathrm{e}}=0.07\right]$. However, the interaction between test and serial position showed a significant quadratic trend in a polynomial test of order $\left[F(1,70)=5.65, M S_{\mathrm{e}}=0.07\right]$. There was therefore no difference in overall performance between the implicit and explicit tests. There was also no overall difference in performance across the serial positions of studied target items associated and recalled, but the implicit and explicit tests differed in the distribution of these serial positions. A reexamination of Figure 1 shows that quadratic trends in the two tests went in opposite directions. There was no indication of a primacy effect in the implicit test, but there was a significant difference between Serial Positions $1-3$ and $7-9[t(35)=$ $2.08, S E M=0.07]$ in the explicit test, indicating an extended primacy effect. The only evidence of any recency effect was in the explicit test, in which a comparison between Serial Positions 7-9 and 16-18 was significant $[t(35)=2.09, S E M=0.05]$, indicating an extended recency effect.

Weakly related word pairs. A further $2 \times 6$ ANOVA with one between-subjects factor, test, and one withinsubjects factor, serial position, was performed for weakly related word pairs. Probabilities of baseline performance and incorrect recall were subtracted from each data point prior to the analysis. Both the main effects of test $[F(1,70)$ $\left.=12.14, M S_{\mathrm{e}}=0.25\right]$ and serial position $[F(5,350)=$ $\left.2.31, M S_{\mathrm{e}}=0.08\right]$ were significant, but the interaction between test and serial position was not $[F(5,350)=1.05$, $\left.M S_{\mathrm{e}}=0.08\right]$. There was therefore a difference in overall performance between the implicit and explicit tests and an overall difference in performance across the serial positions of studied target items associated and recalled in both tests. Comparisons between Serial Positions 1-3 and 4-6 showed a significant difference in the explicit test $[t(35)=2, S E M=0.07]$, but not in the implicit test $[t(35)=0.39, S E M=0.07]$, indicating that only the explicit test showed a primacy effect. However, in Table 1, the implicit test appears to show a single item primacy effect, and this was confirmed in a comparison between the probability of studied target word association between Serial Position 1 and Serial Positions 2-6 $[t(35)=2.64$, $S E M=0.07]$. Further comparisons between Serial Positions 13-15 and 16-18 were not significant in the explicit test $[t(35)=0.14, S E M=0.07]$ or in the implicit test $[t(35)=0.39, S E M=0.07]$. Neither test therefore ap- 
peared to show a short-term recency effect. Nevertheless, a $2 \times 5$ polynomial test of order between test and nonprimacy serial positions showed a significant linear trend $\left[F(1,70)=10.79, M S_{\mathrm{e}}=0.08\right]$, with no interaction between test and nonprimacy serial positions $[F(1,70)=$ $\left.0.73, M S_{\mathrm{e}}=0.08\right]$. When primacy items were excluded, there were extended recency effects throughout the course of both tests.

\section{GENERAL DISCUSSION}

The main experimental findings were that only weakly related word pairs showed primacy and extended recency effects in primed free association, whereas both weakly and strongly related word pairs showed primacy and extended recency effects in associative cued recall.

A possible explanation for the differences in primacy effects between the tests is based on the premise that only weakly related word pairs require associative connections between the two words to be established or strengthened at study; strongly related word pairs already have a unitized representation in memory that does not require additional strengthening. The establishment or strengthening of associative connections between weakly related word pairs is enhanced for the first word pairs studied because binding of these word pairs benefits from more rehearsal (elaborative or rote) and more focused attention than the remaining word pairs, the current explanations for primacy effects (Atkinson \& Shiffrin, 1968; Craik \& Lockhart, 1972; Glenberg et al., 1980; Rundus, 1971). This enhancement of the connection between the first word pairs studied contributes to both implicit and explicit test performance and may be entirely responsible for the primacy effect in the implicit test. However, the primacy effect in the explicit test does not solely depend on the enhancement of the encoded connection between the first few word pairs studied. If it did, the explicit test would not have shown a primacy effect for strongly related word pairs that did not require strengthening of associative bonds, because they already have unitized memory representations. The primacy effect for strongly related word pairs in the explicit test must therefore depend on the involvement of intentional retrieval strategies at test and, by implication, the primacy effect for weakly related word pairs in the explicit test depends on two factors, the enhancement of the connection between the first word pairs studied and the involvement of intentional retrieval strategies at test.

This explanation is consistent with the absence of primacy effects in word stem completion but the presence of primacy effects in word stem cued recall (Brooks, 1994). No associative connections were required to be made when the single word stimuli were being encoded, but intentional retrieval strategies still resulted in a primacy effect in the explicit test, probably because the first few list items benefited from deeper encoding resulting from more rehearsal and more focused attention than the remaining items, the current explanations for primacy effects (see, e.g., Atkinson \& Shiffrin, 1968; Craik \& Lockhart, 1972).

The differences in extended recency effects between the tests may be because the encoded strengthened associative connections between weakly related word pairs were susceptible to decay or interference during both the implicit and the explicit tests. This would account for all the extended recency effect in the implicit test, since no measurable effect occurred with strongly related word pairs. However, the involvement of intentional retrieval strategies at test also contributed to the extended recency effect in the explicit test, since there was some indication of an effect with strongly related word pairs.

It is difficult to relate the results of this study to the current theoretical issue of whether dissociations between implicit and explicit memory tests reflect retrieval from different memory systems (see, e.g., Schacter \& Tulving, 1994) or whether they reflect different degrees of overlap between encoding and test processes within a unitary memory system (see, e.g., Roediger \& Srinivas, 1993) because neither theory makes any predictions about serial position effects. However, a current development in the systems versus processing debate is that "a components-of-processing" approach is better able to account for functional dissociations between different perceptual tests and between different conceptual tests, which pose problems for both systems and processing theories (see Roediger, Buckner, \& McDermott, 1999). The components-of-processing theory is based on ideas from Moscovitch and his colleagues (e.g., Moscovitch, 1994) that task performance typically involves a number of different components and that tasks differ as to the specific components involved. Within this approach, two tasks, or even the same task using different stimulus items, may have a number of processing components in common, but other processing components that are unique. In the present study, encoding weakly related word pairs may have utilized an additional processing component that was not required when strongly related word pairs were encoded. Similarly, explicit retrieval of strongly related word pairs may have utilized an additional processing component to implicit association of the same word pairs.

The present paper indicates that there is still some scope for examining the serial positions of items retrieved to enhance our knowledge and understanding of the systems and processes that contribute to human memory.

\section{REFERENCES}

Atkinson, R. C., \& Shiffrin, R. M. (1968). Human memory: A proposed system and its control processes. In K. W. Spence \& J. T. Spence (Eds.), The psychology of learning and motivation: Advances in research and theory (Vol. 2, pp. 89-195). San Diego: Academic Press.

BOWERS, J. S., \& SCHACTER, D. L. (1990). Implicit memory and test awareness. Journal of Experimental Psychology: Learning, Memory, \& Cognition, 16, 404-416.

BroOKS, B. M. (1994). A comparison of serial position effects in implicit and explicit word-stem completion. Psychonomic Bulletin \& Review, 1, 264-268.

Craik, F. I. M. (1970). The fate of primary items in free recall. Journal of Verbal Learning \& Verbal Behavior, 9, 143-148. 
CRAIK, F. I. M., \& LockHART, R. S. (1972). Levels of processing: A framework for memory research. Journal of Verbal Learning \& Verbal Behavior, 11, 671-684.

DiAmond, R., \& Rozin, P. (1984). Activation of existing memories in anterograde amnesia. Journal of Abnormal Psychology, 93, 98-105.

Gershberg, F. B., \& Shimamura, A. P. (1994). Serial position effects in implicit and explicit tests of memory. Journal of Experimental Psychology: Learning, Memory, \& Cognition, 20, 1370-1378.

Glanzer, M., \& CUNITZ, A. R. (1966). Two storage mechanisms in free recall. Journal of Verbal Learning \& Verbal Behavior, 5, 351-360.

Glenberg, A. M., Bradley, M. M., Stevenson, J. A., Kraus, T. A., Tkachuk, M. J., Gretz, A. L., Fish, J. H., \& Turpin, B. M. (1980). A two-process account of long-term serial position effects. Journal of Experimental Psychalogy: Human Learning \& Memory, 6, 355-369.

GraF, P., \& MANDLER, G. (1984), Activation makes words more accessible, but not necessarily more retrievable. Journal of Verbal Learning \& Verbal Behavior, 23, 553-568.

JENKINS, J. (1970). The 1952 Minnesota word association norms. In L. Postman \& G. Keppel (Eds.), Norms of word association (pp. 9 . 38). New York: Academic Press.

MoscoviTcH, M. (1994). Memory and working with memory: Evaluation of a component process model and comparison with other models. In D. L. Schacter \& E. Tulving (Eds.), Memory systems 1994 (pp. 369-394). Cambridge, MA: MIT Press.

Richardson-Klavehn, A., Gardiner, J. M., \& Java, R. I. (1994). Involuntary conscious memory and the method of opposition. Memory, 2, 1-29.

Richardon-Klavehn, A., Lee, M. G., Joubran, R., \& Biork, R. A. (1994). Intention and awareness in perceptual identification priming. Memory \& Cognition, 22, 293-312.
RoEdiger, H. L., III, Buckner, R., \& MCDERMott, K. B. (1999). Components of processing. In J. K. Foster \& M. Jelicic (Eds.), Memory: Systems, process, or function? (pp. 31-65). New York: Oxford University Press.

RoEDiger, H. L., III, \& MCDermott, K. B. (1993). Implicit memory in normal human subjects. In F. Boller \& J. Grafman (Eds.), Handbook of neuropsychology (Vol. 8, pp. 63-131). Amsterdam: Elsevier.

RoEdiger, H. L., III, \& SRINIVAS, K. (1993). Specificity of operations in perceptual priming. In P. Graf \& M. E. J. Masson (Eds.), Implicit memory: New directions in cognition, development, and neuropsychology (pp. 17-48). Hillsdale, NJ: Erlbaum.

Rundus, D. (1971). Analysis of rehearsal processes in free recall. Journal of Experimental Psychology, 89, 63-77.

RYBASH, J. M., \& OSBORNE, J. L. (1991). Implicit memory, the serial position effect, and test awareness. Bulletin of the Psychonomic Society, 29, 327-330.

SCHACTER, D. L., \& MCGLYNN, S. M. (1989). Implicit memory: Effects of elaboration depend on unitization. American Journal of Psychology, 102, 151-181.

SCHACTER, D. L., \& Tulving, E. (1994). What are the memory systems of 1994? In D. L. Schacter \& E. Tulving (Eds.), Memory systems 1994 (pp. 1-38). Cambridge, MA: MIT Press.

Tulving, E. (1962). Subjective organization in free recall of "unrelated" words. Psychological Review, 60, 344-354.

(Manuscript received March 26, 1998; revision accepted for publication October 6, 1998.) 